\title{
Shifting towards Proactive OHS Risk Management in Romanian Organizations: Systematic Benchmarks
}

\author{
Roland Iosif Moraru $^{1^{*}}$, Gabriel Bujor - Băbuţ ${ }^{1}$, Mihai Popescu - Stelea ${ }^{1}$ and Zoltan-Gyozo \\ Szabo ${ }^{1}$ \\ ${ }^{1}$ University of Petroşani, Faculty of Mines, 332006 Petroşani, Romania
}

\begin{abstract}
As they are anticipative, proactive, and treating assessment as a quasi-continuous phase of risk management, the next step in keeping workers safe seems to be the proper implementation of the guidelines on risk assessments included in ISO 45001. They are indeed inspiring for organizations worldwide to consider risk management as really important, good for the business, and imperative for worker health and safety. What should the Romanian organizations know and do in this spirit? The main objective of this paper is to answer systematically and in a structured way the question of whether there is a set of principles, means to follow, recommendable approaches that, taking into account the general practice established at national level at this time of evolution, allow even to facilitate the transition from reactive to proactive management of occupational hazards, with all the advantages and - at the same time challenges that this change implies? The main possible answers to this question are investigated, resulting in a set of conclusions with practical applicability, which can guide the process of streamlining the results of accidents at work and occupational diseases prevention work at the national level, within a coherent and unitary vision.
\end{abstract}

\section{Introduction}

In Romania, the European Union has had the greatest impact on OSH activity, imposing a solid legal framework covering a wide variety of occupational hazards and a significant number of regulations. Thus, workers have the right to a high level of OSH, as well as the right to a work environment adapted to their professional needs and to allow them professional activity for as long as possible [1].

On 25.05.2017, EU-OSHA published the report "Risks to health and safety at work: a joint analysis of three major surveys" which presents the main conclusions drawn from a joint analysis of the ESENER-2 study (2019), the results of the 2013 Eurostat LFS survey on accidents at work and the results of health problems highlighted by the 2015 Eurofound EWCS survey. „The aim was to have an overview of the state of OSH in Europe, bringing

\footnotetext{
* Corresponding author: roland moraru@yahoo.com
} 
together, on the one hand, the perspectives of organizations on risk management and risk awareness, and on the other hand, the perspective and perceptions of workers exposed to risks, but also the results of the implementation of the OSH strategy". In summary, this report highlights recommendations that future action should be based on efforts to strengthen [2]:

a) The commitment of the organizations leadership regarding the specific management of OSH risks and musculoskeletal disorders;

b) Psycho-social risk management;

c) Official representation of employees, with involvement in the analysis of findings of work accidents/incidents, design and configuration of measures for improvement and / or prevention in the field of OSH, as well as in the case of developing risk assessment tools;

d) Promoting and respecting safe work environments and workers wellbeing;

e) Increasing resources for risk management in enterprises.

A strategic leadership perspective on OSH issues related to the organization's mission can establish a context of growth, profitability, efficiency, effectiveness in addition to generating value for customers and for workers in terms of their safety and health at work [3]. This can not be achieved without a shift towards a proactive management of OHS in any Romanian organization.

On the other hand, article $7, \S 4$, letters a) and b) together with article 12 , $\S 1$, letter a) of the Occupational Safety and Health Act place the general duty on any employer to conduct their business in such a manner that ensures, as far as is reasonably achievable, that both employees and contractors/visitors who may be affected thereby are not exposed to risks to their safety and health [4].

A large spectrum of other legal acts, both general and industry branch specific, include the general requirement for all employers to assess the risks to employees and any others who may be affected by their activities, to enable them to identify measures necessary to comply with their duties under occupational safety and health law [5].

Therefore, risk assessment is recognized as an integral/basic stage of successful/effective management of workers health and safety [6]. A meaningful and powerful OHS management system will depend, amongst some other relevant aspects, on adapted, suitable and proficient risk assessments being carried out and on proper implementation of risk reduction measures [7].

The results of the risk assessment can/should be valorised to ground decisions as to whether any existing safety barriers are adequate, or whether additional ones are likely to offer additional confidence in attaining the preset safety objectives. Risk assessment, as a risk management basic step (but also as a powerful tool itself) can also be a support in performing a systematic comparison of different risk control/reduction options so that the optimal multi-criteria decision can be adopted. These considerations are standing valid in the present Romanian OHS management practices, when the need to really make the leap towards the proactive safety management becomes more and more imperative [8].

Several specific errors, drawbacks and traps that continue for more than 10 years to strike, in Romania, the results effectiveness of a process that continues to be unacceptably frequently characterized by an unpractical formalism were highlighted in previous research [9]. In this context, the present paper tries to synthesize the basic principles and recommended occupational risk control practices, outlining the way in which the hazard reduction and risk mitigation strategies should be founded on the involvement of all stakeholders, on a participatory approach, on positive safety culture and behaviour, namely on proactive safety management.

\section{A Glimpse on Proactive Safety from ISO 45001 Perspective}


For any organization that is already OSHAS 18001 certified and are looking forward to migrate towards to ISO 45001 [10], there can be extracted several basic changes and additions from the new standard which need to be considered in the future (see Figure 1):

- Planning elements - There are included in the new framework supplementary details regarding how should be addressed risks; besides documentation requirements, from the proactive approach it has a growing importance to give a greater to the component of how is impacted the OSH planning by the workers involvement and participation.

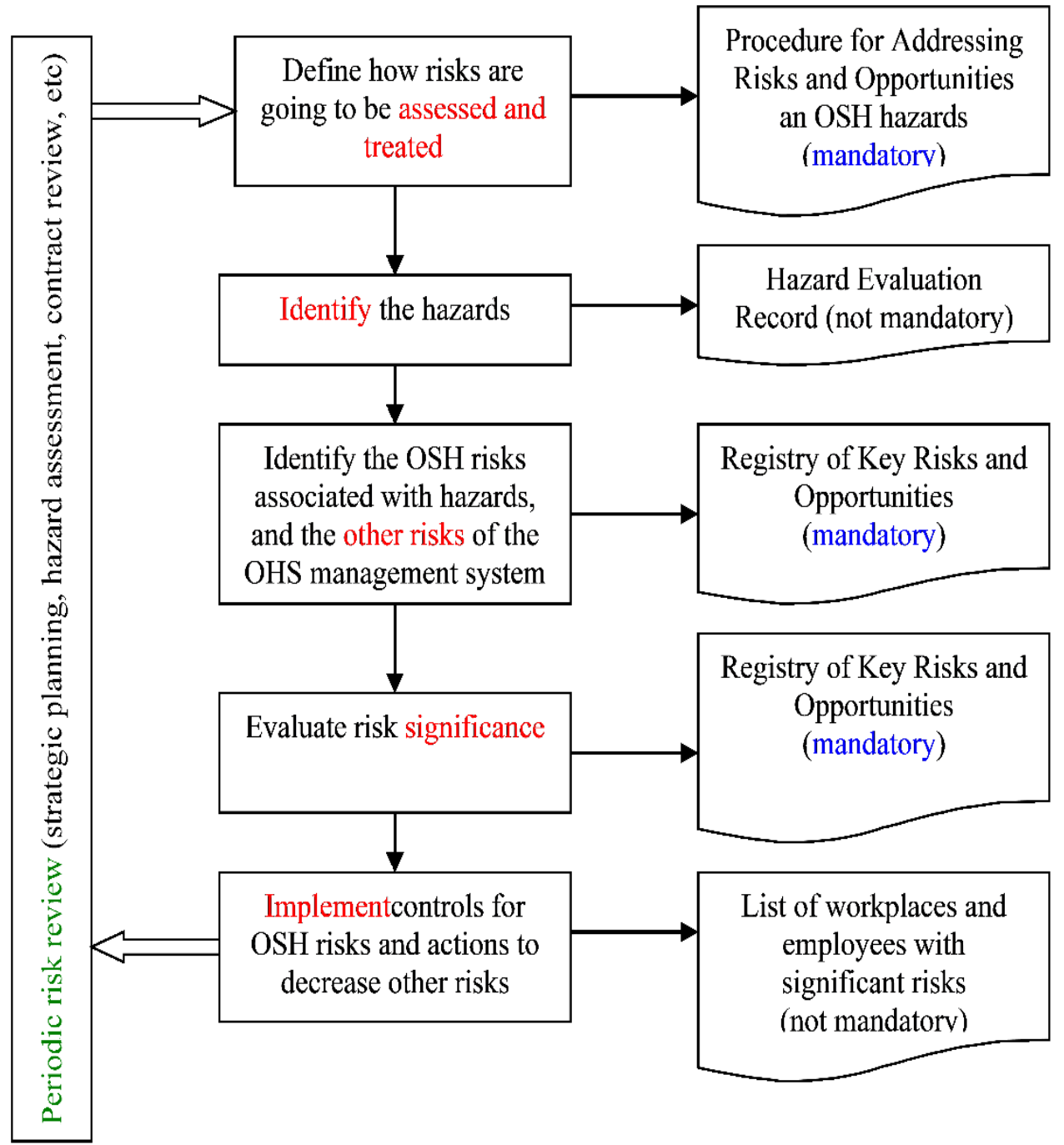

Fig. 1. Diagram of ISO 45001 Risk Management Process (adapted from Hammar, [11])

- $\quad$ Risk and opportunity identification - Organizations are required to establish the entire relevant internal and external context issues (in the spirit of ISO 31000; 2018 standard), likely to exert significant impact on achieving the envisaged OSH objectives and outcomes; this should include considerations regarding contractors 
or anything else could impact the OSH capabilities, with special focus on the opportunities to improve the OSH management system.

- Extended field of risk scoping, assessment and treatment - meaning that changes in the world of work sharply require the extension of the risk approach from the "classical" ones (physical, chemical, biological) to the components of workers well-being, taking into account emerging risks, psychological demands, moral or other harassment, bullying, violence at work, diseases caused by occupational stress

- OSH competencies of workers - new specifications have been introduced that explicitly require organizations to ensure, determine and monitor the OSH competencies and abilities of workers; be able to know the risks, to identify the new ones, to know how to follow the procedures and to take the appropriate actions in case of need.

If there are organizations or stakeholders who - being optimistic - hope to be provided with a clear plan on how to implement all these new ideas in the field of occupational safety and health management, it is somewhat disappointing to be in no such case will be found in ISO 45000. The standard imposes requirements, but leaves it to the discretion of each economic organization to assess the risk to the business, the periodicity of the risk assessment, as well as all other practical aspects of management.

The positive consequence - and should be interpreted as such - is related to the generous flexibility offered to the field of action. It is certainly obvious that various types of companies and, respectively, specific fields of activity (from technology, manufacturing, oil and gas, IT, agriculture, etc.) face particular risks, are located in particular contexts, being characterized of individuality and uniqueness in a general contextual landscape [12].

To complicate matters further, the standard clearly states two risks categories to be assessed, so what will be the most appropriate risk management methodology? Clause 6.1.2, regarding the identification of hazard and risks and opportunities assessment, gives consideration - as mentonned before - to two different risk categories which should be considered within the OSH management system, as follows:

- Category 1 - Hazard identification: the first clause, 6.1.2.1, requires to give consideration to the hazards/risks which are usually present in the processes of any organization., but, besides the regular conditions, also should be enviseged the transitory states including possible emergency situations requiring prompt and effective responses, situations triggered by factors such as changes in the activities, processes and OSH management system.

- Category 2 - OSH Risk assessment: the second risk category can be found in clause 6.1.2.2, which refers not only to the risks related to OHS hazards, but also to other new risks having the potential to affect the OHS management system, issued from the internal and external context and identified earlier in the standard. These are ranging from changes in the legal and regulatory requirements, to the needs of stakeholders/interested parties.

- It is required that for both risk categories the assessment to be defined, proactive rather than reactive, used systematically, and documented.

In short, for all activities, processes and work areas, it is necessary to identify the existing hazards in the workplace, to identify all categories of staff that may be involved / affected (including mandatory - taking into account contractors and visitors). After the identification phase of the hazards, follows the stage of identification of the concrete forms of manifestation of the associated risks, their hierarchy and the establishment of the means of prevention / protection for the reduction / control of the highlighted risks. Representing the basic stage of the process, this assessment of the hazards and risks induced by the 
organisation's activities remains a critical part of what is needed to improve occupational health and safety performance [13].

The new problem with the OSH management system is that it will have to establish, implement and update a procedure for the continuous identification of hazards, risk assessment and determination of prevention / protection measures. Routine and non-routine activities, human behavior and capabilities, the organisation's legal compliance obligations, infrastructure and equipment / materials in the workplace will be further included in this assessment [14-16].

Hazard identification should be a proactive process that allows risks to be prioritized and documented, as a key step in their subsequent effective treatment. Given that this phase has an overwhelming role on the performance and efficiency of the OSH management system and - implicitly - on the well-being of workers, by minimizing accidents, incidents and all other categories of undesirable events, it is clear that this process it must be taken very seriously.

In Romania, not infrequently, things proceed in a somewhat formal, devoid of manner, without pursuing continuous improvement based on prioritization and proper allocation of resources. As a result, a major shift towards a proactive approach from this perspective, as opposed to reactive or even pathological management (in Hudson's sense), is required. Furthermore, many elements of change in the perspective and - itself - of the culture of approaching risk assessment and OSH management should occur urgently in our country, involving the real participation of workers for continuous improvement.

\section{A Conceptual Model for the Major Vault in Romanian Organizations: Switching to a Proactive OHS Management Pattern}

Briefly, proactive safety measures necessitate the reliance on any strategy/tactic likely to be employed in order to minimize the likelihood of an undesired event taking place. Simply said, being involves anticipating accidents/incidents/losses, being prepared, minimizing response times, minimizing the number of injuries/illnesses that can occur in the workplace, and consequently, dramatically decreasing the number and amount of worker's compensation claims.

Proactive safety requires, amongst others, to have in place an operational system able to learn all employees to gain and use experience in risk identification and/or assessment, at their own level of competencies. Various jobs and workplaces from differing industries are characterized - each of them - by unique spectrums of accident and occupational illness risk. It is of paramount importance that any worker to be knowledgeable and efficient at identifying/assessing/mitigating safety hazards on the job.

The designation "pro" quite literally means "before." Individuals that are proactive, it means that they are striving to make things happen instead of waiting for things to happen to them. When we think about safety management, being proactive entails the use of safety solutions that are designed to prevent an undesired event before his occurrence.

In comparison, reactive safety measures are essentially quite the opposite. A largely perceived advantage of operating under this approach is related to resource saving. However, both the direct and indirect costs related to losses generated by workplace injuries and other costs induced by materialized accident scenarios are notable, sometimes even onerous.

Proactive OHS management should focus on how daily performance usually succeeds rather than on why it fails from time to time, and actively try to improve the anticipation of possible failures rather than simply minimizing the occurrence rate and severity of the 
future undesired events. This kind proactive OHS management requires that adjustments are performed before anything undesired happens rather than after. The basic advantage of this approach is related to the fact that interventions can be implemented in time, or sometimes even in very early stages (such as the design phase of a work system), the preventive vector being thus favored.

Another feature is that early safety interventions, in general, require significantly less effort (costs) because the consequences of the any considered undesired event (work accident, technical failure, human error, major accident etc) will have had less time to develop and expand. Also, it should be emphasized that any early detection and intervention is likely to save valuable time and -thus - important resources.

Fig. 2 presents a systematic approach to proactive OHS risk management, illustrating the recommended hierarchical relationship among inherent, engineered, and procedural safety measures and barriers. An inherently safer work system, by the very way it is designed, induces/generates lower severity or no consequences in the event of an accident occurred. The basic principles of inherent safety outline various techniques to obtain an inherently safer work system four of the most general and widely applicable principles being briefly discussed bellow:

i. minimization through design; this principle involves risk reduction by employing smaller amounts of hazardous substance/material/agent or performing a risky procedure fewer times within a given industrial process.

ii. substitution/replacement; measures are taken with a view to replace a potentially hazardous equipment, material, process, structure route, or procedure with a less hazardous one, thus either totally eliminating or reducing the potential of that hazard to trigger undesired events;

iii. moderation of unavoidable hazards; this principle is aimed at using hazardous materials (when their cannot be eliminated and/or minimized) under less hazardous conditions or in a less hazardous form. Moderation is, no doubt, the most difficult inherent safety principle to visualize and, consequently, to implement into practice. Minimization of fuel loads, replacement of less hazardous chemical agents if possible, and simplification of equipment for bulksolids handling are perhaps conceptually more familiar to industrial safety engineers. In most of the cases when moderation is envisaged, it is often with a view to diminishing energy levels involved during processing by means of a reduction in temperature, concentration, flow or pressure. Such reductions can sometimes be targeted and achieved by use of an adequate catalyst in applications of reaction engineering.

$i v$. simplification/back-up safety measures optimization. In this fourth applicable principle, the main goal is to design processes and related equipment to reduce the opportunities for errors by eliminating excessive use of back-up safety features and add-on protective devices.

However, in general, Fig. 2 indicates that there are several opportunities for reducing risk through proactive safety when the basic principles of inherent safety are applied early in the engineering design cycle. Optimal application of these principles systematically facilitates the reduction of both consequences severity and likelihood of occurrence before taking the next important step, namely the addition of engineered safety measures and the development/implementation of safe work procedures (Standard Operating Procedures). 


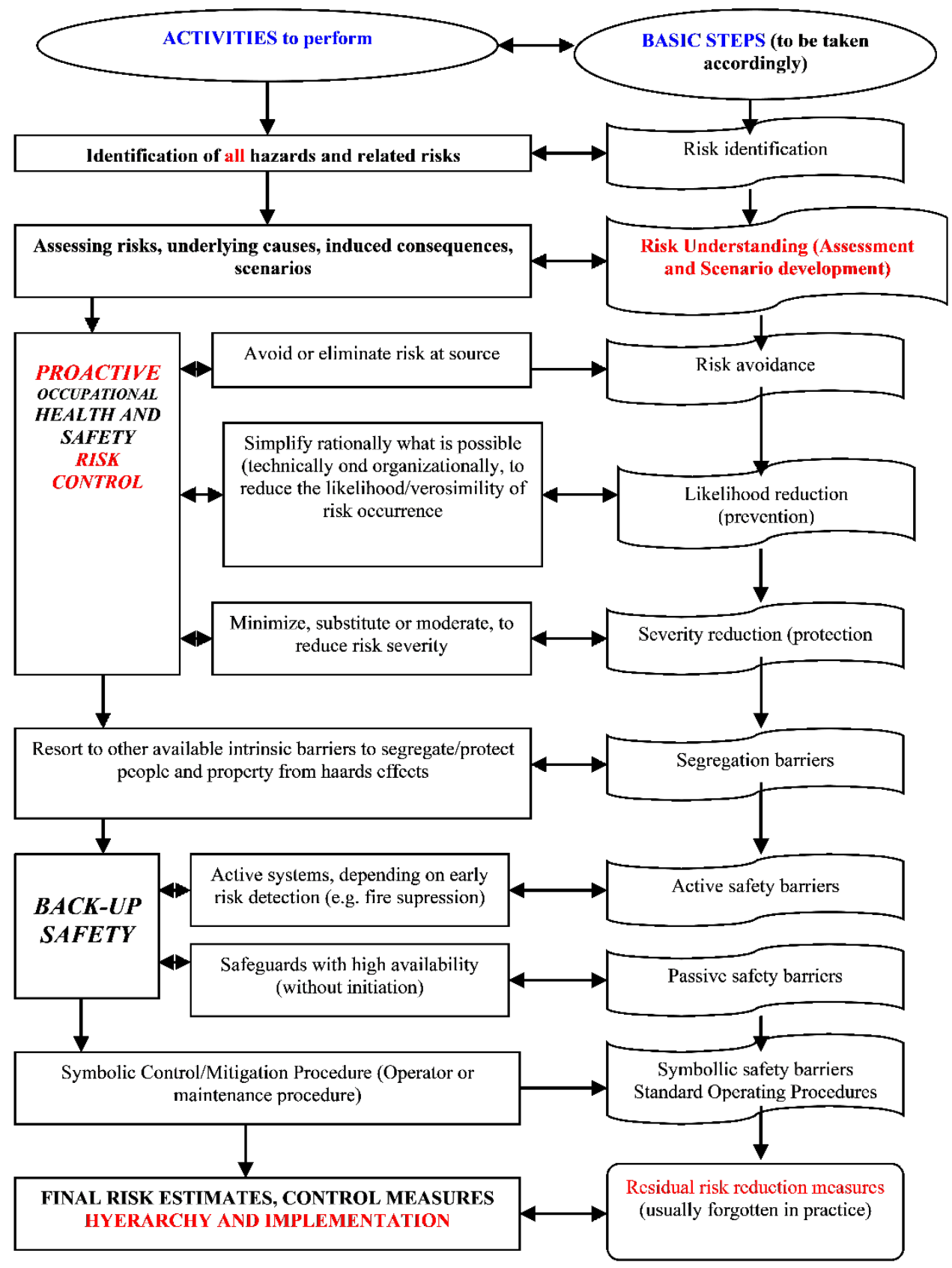

MONITORING AND REVIEW

(safety inspections, regular auditing, ongoing training, near-miss reporting and active supervision)

Fig 2. The recommended systematic approach of proactive OHS risk management 
An authentic proactive safety management vision and practice in Romanian organizations involves the need for several efforts to be invested at top level management of any organization - to anticipate what is likely to occur, to prepare adequate risk treatment plans and to really implement them, to allocate accordingly financial, material, human and other required resources and to develop well-documented, realistic and feasible contingency plans.

\section{Conclusions}

The conclusions summarized following the analysis performed in the paper have the potential to be a benchmark of good practice for Romanian organizations aimed at improving their performance in the field of OHS and their overall effectiveness, and can be formulated as follows:

1. Focusing on usual/regular events and selecting events based on their likelihood rather than their severity (magnitude of negative consequences) is, in general, very advisable. Preventive behavior is much easier to implement at all levels (workers, line and top management) for events that occur frequently and regularly, than in the case of those with rare and irregular occurrence. Certainly, small quasicontinuous improvements in daily performance will generate more important results than a single major improvement, even with an exceptional result. This tactic of materializing the proactive approach will also generate lower implementation costs, while facilitating the establishment and development of a positive workplace safety culture.

2. Taking time enough for reflection, learning, communication, consulting and understanding the root causes and all the various other aspects involved (usually complex in their nature and manifestation) is mandatory. A general risk assessment, followed by a general prevention and protection plan, without substance and concreteness, will never be enough. It will always be more effective to consolidate by reflection the experience gained, to refresh resources, in the sense of increasing the degree of learning and organizational resilience. Without an adequate and consistent culture of resource allocation (including time), all these pillars of the improvement process will be weak, and even a false sense of risk control can be established in the organization. Not infrequently, this is the "de facto" situation present in Romanian companies that go on the line of minimum resistance of compliance with the OSH legislative provisions.

3. The fact that failure is possible should never be forgotten or neglected. The prospective elaboration of a preliminary (but always updated) list of potential dysfunctions and the attempt to imagine how they can occur (the development of event scenarios) is currently an almost completely neglected practice. This is the very essence of the proactive OSH management, allowing the adoption of fair decisions on the appropriate allocation of resources for prevention and protection barriers real implementation.

4. Because the pressure of economic results mainly favors economic efficiency, in relation to the occupational safety and health component, both the proactive and the reactive component are present in the real world of work. Usually, the latter is favored, which diminishes the chances of being proactive. Therefore, attention must be paid to things that go well, as well as those that go wrong, learning equally from everything, even from neutral situations, but correctly understanding what is happening. We know very well that things do not happen perfectly only because the worker follows a procedure, but also that he makes certain adaptations to the situation, from which we can extract special lessons. For this, however, the 
proactive safety approach must be completed with a functional participatory management style.

5. The future is uncertain and the predictions are inaccurate. For proactive OHS management to work, it is necessary to provide with an acceptable degree of certainty and to have adequate means of response. Furthermore, a correct understanding of the work system, of all its components and of the way in which they are interconnected will be necessary.

6. Emphasizing behavioral patterns and relations across events/scenarios rather than individual risk factors is likely to enlarge and deepen the knowledge and understanding the answers given to the basic questions: Where, who, what, why is happening? Mitigating adverse consequences thus provides a much more solid, stable foundation for decision-making and will facilitate the process of continuous improvement of the organization.

Nevertheless, proactive safety inspections performed on regular basis, auditing periodically, ongoing/continuous effective training, awareness raising, near-miss reporting and investigation and together with active supervision and a participatory management style based on operational communication-consultation activities should all be included a proactive OHS management structure.

\section{References}

1. A. Gaureanu, A. Drăghici, H. Weinschrott,Quality - Access to Success, 20, 141-146, supplement: 1/(2019)

2. I. Houtman, I.Eekhout, , A. Venema, M. B. Roozenboom \& Van Buuren, S. Health and safety risks at the workplace: a joint analysis of three major surveys, p. 12. EU-OSHA https://doi.org/10.2802/835406, Accesed: 2 February 2021 at: https://osha.europa.eu/en/toolsandpublications/publications/health-and-safety-risks-workplace-joint-analysis-threemajor/view (2017)

3. A. Gaureanu, A. Drăghici, A. Mateescu, H. Weinschrott, „Gaps And Directions To Improve Occupational Safety And Health Training Programs In Romania" ICPR-AEM in 4th QIEM, Cluj Napoca, Romania, July (2016).

4. Romanian Parliament (2006). The 319/2006 Act on Occupational Health and Safety, Oficial Monitor of Romania, Part I, nr. 646/26.07 (2006)

5. Romanian Government, Government Decision no. 1146/2006 concerning the minimum safety and health requirements for the use of work equipment by workers at work (in Romanian), The Official Journal of Romania, Part I, no. 815/03.10.2006 (2006)

6. G.B, Băbuţ, R.I., Moraru, Occupational risk assessment framework in Romania: an institutional perspective, $16^{\text {th }}$ SGEM 2016, Albena, Bulgaria, 30.06-06.07, (2016)

7. L.I., Cioca, R.I., Moraru , G.B., Băbuţ, A Framework for Organizational Characteristic Assessment and their Influences on Safety and Health at Work, $15^{\text {th }}$ KBO ConferenceInternational Scientific Conference ,, The Knowledge Based, Land Forces Academy Sibiu, Romania (2009)

8. L. Ivaşcu, L.I., Cioca, Opportunity Risk: Integrated Approach to Risk Management for Creating Enterprise Opportunities, $2^{\text {nd }}$ International Conference on Psychology, Management and Social Science, Psychology, Management and Social Science, Shenzhen, China, Book Series: Advances in Education Research, Volume 49, pp.77-80 (2014)

9. R.I. Moraru, G.B. Băbuţ, L.I. Cioca, Drawbacks and traps in risk assessment: examples in Romania, 5th MSE, Vol. 2, Sibiu, Romania (2011)

10. International Organization for Standardization, ISO 45001: 2018 Standard, Occupational health and safety management systems - Requirements with guidance for use, (2018)

11. M. Hammar, What to include in risk management methodology according to ISO 45001:2018, March 21, 2019, Accessed: 5 2021, https://advisera.com/45001academy/blog/2019/03/21/what-to-include-in-risk-managementmethodology-according-to-iso-45001/, (2019) 
12. R.I. Moraru, G.B. Băbuț, L.I. Cioca, Study of methane flow in caved goafs adjacent to longwall faces in Valea Jiului coal basin, 13th SGEM, I, 731-738 (2013)

13. ASRO, SR Guide ISO 73: 2010 - Risk management. Vocabulary (in Romanian), Romanian Standards Association (ASRO), Bucharest, Romania, (2010)

14. R.I. Moraru, G.B.Băbuț, Quality - Access to Success Journal, 11, 4, 50-59 (2010)

15. L. Ivaşcu, L.I., Safety, 5 (1), Article Number: 12, (2019)

16. R.I., Moraru, G.B., Băbuț, L.I., Cioca, Knowledge management applications in occupational risk assessment processes, $17^{\text {th }} \mathrm{KBO}$ - The Knowledge-Based Organization: Management and Military Sciences, pp. 735-740, Sibiu, Romania (2011) 